Pure Mathematical Sciences, Vol. 3, 2014, no. 3, 121 - 127

HIKARI Ltd, www.m-hikari.com

http://dx.doi.org/10.12988/pms.2014.4614

\title{
The Convergence between Approximate Solutions and the Unique Solutions of SDEs
}

\author{
Wenfei Xu \\ College of Mathematics and Information Science \\ Henan Normal University, Henan Province, 453007, China \\ Shanshan Chen \\ College of Mathematics and Information Science \\ Henan Normal University, Henan Province, 453007, China \\ Copyright (C) 2014 Wenfei Xu and Shanshan Chen. This is an open access article dis- \\ tributed under the Creative Commons Attribution License, which permits unrestricted use, \\ distribution, and reproduction in any medium, provided the original work is properly cited.
}

\begin{abstract}
In this paper, we focus on Euler-Maruyama's approximate solutions of SDEs. Thus, we will be able to show that the unique solution $x(t)$ of the stochastic differential equation converge to approximate solution.
\end{abstract}

Mathematics Subject Classification: $60 \mathrm{H} 10$

Keywords: Stochastic differential equation, Euler-Maruyama's approximate solutions, Convergence

\section{Introduction}

Nowadays, we know that not all the solutions of stochastic differential equations have the explicit expressions. Thus, we often attempt to seek for approximate solution rather than the accurate solution, such as Euler-Maruyama's approximate solutions. Of course, there exist amounts of conclusions between approximate solutions and the unique solutions of SDEs. Based on these facts, we will be able to show that the unique solution $x(t)$ of the stochastic differential equation converge to Euler-Maruyama's approximate solution in this paper. 
Firstly, we will give some preconditions. Let $(\Omega, \mathrm{F}, P)$ be a complete probability space with a filtration $\left\{\mathrm{F}_{t}\right\}_{t \geq 0}$ satisfying the usual conditions. Throughout this paper, unless otherwise specified, we let $B(t)=\left(B_{1}(t), B_{2}(t), \cdots, B_{m}(t)\right)$, $t \geq 0$ be $m$-dimensional Brownian motion defined on the space. Let $0 \leq t_{0}<$ $T<\infty$. Let $x_{0}$ be an $\mathrm{F}_{t_{0}}$-measurable $R^{d}$-valued random variable such that $\mathbb{E}\left|x_{0}\right|^{2}<\infty$. Let $f: R^{d} \times\left[t_{0}, T\right] \rightarrow R^{d}$ and $g: R^{d} \times\left[t_{0}, T\right] \rightarrow R^{d \times m}$ be both Borel measurable. We will consider the $d$-dimensional stochastic differential equation of Itô type

$$
d x(t)=f(x(t), t) d t+g(x(t), t) d B(t) \text { on } t_{0} \leq t \leq T
$$

with initial value $x\left(t_{0}\right)=x_{0}$.

Secondly, let us illustrate the definition of Euler-Maruyama's approximate solutions. They are defined as follows: For every integer $n \geq 1$, define $x_{n}(t)=$ $x_{0}$, and then for $t_{0}+(k-1) / n<t \leq\left(t_{0}+k / n\right) \wedge T, k=1,2 \ldots$,

$$
\begin{aligned}
x_{n}(t) & =x_{n}\left(t_{0}+\frac{k-1}{n}\right)+\int_{t_{0}+(k-1) / n}^{t} f\left(x_{n}\left(t_{0}+\frac{k-1}{n}\right), s\right) d s \\
& +\int_{t_{0}+(k-1) / n}^{t} g\left(x_{n}\left(t_{0}+\frac{k-1}{n}\right), s\right) d B(s) .
\end{aligned}
$$

Note that if define

$$
\hat{x}_{n}(t)=x_{0} I_{\left\{t_{0}\right\}}(t)+\sum_{k \geq 1} x_{n}\left(t_{0}+\frac{k-1}{n}\right) I_{\left(t_{0}+(k-1) / n, t_{0}+k / n\right]}(t),
$$

for $t_{0} \leq t \leq T$. Then from the equations (2) and (3), we can obtain the expression of Euler-Maruyama's approximate solutions as follows:

$$
x_{n}(t)=x_{0}+\int_{t_{0}}^{t} f\left(\hat{x}_{n}(s), s\right) d s+\int_{t_{0}}^{t} g\left(\hat{x}_{n}(s), s\right) d B(s) .
$$

For the Euler-Maruyama's approximate solutions, the following results are well-known. Of course, we will list two important conditions before results for convenience:

There exists two positive constants $\bar{K}, K$ such that

1. (Lipschitz condition) for all $x, y \in R^{d}$ and $t \in\left[t_{0}, T\right]$

$$
|f(x, t)-f(y, t)|^{2} \vee|g(x, t)-g(y, t)|^{2} \leq \bar{K}|x-y|^{2},
$$

2. (Linear growth condition) for all $(x, t) \in R^{d} \times\left[t_{0}, T\right]$

$$
|f(x, t)|^{2} \vee|g(x, t)|^{2} \leq K\left(1+|x|^{2}\right)
$$


Theorem 1.1 [2] Under the linear growth condition (6), for all $n \geq 1$, we have

$$
\sup _{t_{0} \leq t \leq T} \mathbb{E}\left|x_{n}(t)\right|^{2} \leq C_{1}:=\left(1+3 \mathbb{E}\left|x_{0}\right|^{2}\right) e^{3 K\left(T-t_{0}\right)\left(T-t_{0}+1\right)}
$$

and for $t_{0} \leq s<t \leq T$ with $t-s \leq 1$

$$
\mathbb{E}\left|x_{n}(t)-x_{n}(s)\right|^{2} \leq C_{2}(t-s),
$$

where $C_{2}=4 K\left(1+C_{1}\right)$.

Theorem 1.2 [3, Theorem 4.3 in Section 2.4] Let $p \geq 2$ and $x_{0} \in L^{p}\left(\Omega, R^{d}\right)$. Assume that the linear growth condition (6) holds and $x(t)$ is the unique solution of the equation (1). Then there exists a positive constant $C=C(T, K)$ such that

$$
\mathbb{E}|x(t)-x(s)|^{p} \leq C(t-s)^{\frac{p}{2}}, \text { for all } t_{0} \leq t \leq T
$$

where

$$
C=2^{p-2}\left(1+\mathbb{E}\left|x_{0}\right|^{p}\right) e^{p \alpha\left(T-t_{0}\right)}\left(\left[2\left(T-t_{0}\right)\right]^{\frac{p}{2}}+[p(p-1)]^{\frac{p}{2}}\right)
$$

and $\alpha=\sqrt{K}+K(p-1) / 2$.

Based on the above works, the aims of this paper is to discuss that the unique solution $x(t)$ of the stochastic differential equation converge to EulerMaruyama's approximate solution.

\section{Main Results}

\subsection{The convergence between Euler-Maruyama's approx- imate solutions and the unique solution.}

Now we can state our main result.

Theorem 2.1 Let $f(x, t)$ and $g(x, t)$ be continuous. Let $x\left(t_{0}\right)=x_{0} \in$ $L^{p}\left(\Omega, R^{d}\right)$ be a bounded $R^{d}$-valued $\mathrm{F}_{t_{0}}$-measurable random variable. Let the linear growth condition (6) hold. Assume that the equation (1) has the unique solution (that means the pathwise uniqueness) $x(t)$. Then the Euler-Maruyama's approximate solutions $x_{n}(t)$ converge to $x(t)$ in the sense that

$$
\lim _{n \rightarrow \infty} \mathbb{E}\left(\sup _{t_{0} \leq t \leq T}\left|x_{n}(t)-x(t)\right|^{p}\right)=0 .
$$




\subsection{Some Lemmas}

Before giving the proofs of main theorems, we need to show the following some lemmas based on Theorem 1.1.

Lemma 2.2 Under the linear growth condition (6), for all $n \geq 1$ and $p \geq 2$,

$$
\sup _{t_{0} \leq t \leq T} \mathbb{E}\left|x_{n}(t)\right|^{p} \leq C .
$$

where $C$ is a positive constant related to $K, p, T$.

Proof Fix $n \geq 1$ and $p \geq 2$ arbitrarily. Note from equation (4) that for $t_{0} \leq t \leq T$,

$$
\begin{aligned}
\left|x_{n}(t)\right|^{p} & =\left|x_{0}+\int_{t_{0}}^{t} f\left(\hat{x}_{n}(s), s\right) d s+\int_{t_{0}}^{t} g\left(\hat{x}_{n}(s), s\right) d B(s)\right|^{p} \\
& \leq 3^{p-1}\left|x_{0}\right|^{p}+3^{p-1}\left|\int_{t_{0}}^{t} f\left(\hat{x}_{n}(s), s\right) d s\right|^{p}+3^{p-1}\left|\int_{t_{0}}^{t} g\left(\hat{x}_{n}(s), s\right) d B(s)\right|^{p} .
\end{aligned}
$$

Using the Hölder inequality, the martingale inequality [Lemma 2.4] and the definition of Euler-Maruyama approximate solutions, we can then derive that

$$
\begin{aligned}
\mathbb{E}\left|x_{n}(t)\right|^{p} & \leq 3^{p-1} \mathbb{E}\left|x_{0}\right|^{p}+2^{\frac{p-2}{2}}\left[3\left(t-t_{0}\right)\right]^{p-1} K^{\frac{p}{2}} \int_{t_{0}}^{t}\left(1+\mathbb{E}\left|\hat{x}_{n}(s)\right|^{p}\right) d s \\
& +2^{\frac{p-2}{2}} 3^{p-1}\left(\frac{p(p-1)}{2}\right)^{\frac{p}{2}}\left(t-t_{0}\right)^{\frac{p-2}{2}} K^{\frac{p}{2}} \int_{t_{0}}^{t}\left(1+\mathbb{E}\left|\hat{x}_{n}(s)\right|^{p}\right) d s \\
& \leq 3^{p-1} \mathbb{E}\left|x_{0}\right|^{p}+\beta \int_{t_{0}}^{t}\left(1+\sup _{t_{0} \leq r \leq s} \mathbb{E}\left|x_{n}(r)\right|^{p}\right) d s,
\end{aligned}
$$

for all $t_{0} \leq t \leq T$, where $\beta=\frac{1}{6}(18 K)^{\frac{p}{2}}\left(T-t_{0}\right)^{\frac{p-2}{2}}\left[\left(T-t_{0}\right)^{\frac{p}{2}}+\left(\frac{p(p-1)}{2}\right)^{\frac{p}{2}}\right]$. Consequently,

$$
\sup _{t_{0} \leq t \leq T} \mathbb{E}\left|x_{n}(t)\right|^{p} \leq 3^{p-1} \mathbb{E}\left|x_{0}\right|^{p}+\beta \int_{t_{0}}^{T}\left(1+\sup _{t_{0} \leq r \leq s} \mathbb{E}\left|x_{n}(r)\right|^{p}\right) d s .
$$

Since Gronwall inequality implies

$$
1+\sup _{t_{0} \leq t \leq T} \mathbb{E}\left|x_{n}(t)\right|^{p} \leq\left(1+3^{p-1} \mathbb{E}\left|x_{0}\right|^{p}\right) e^{\beta\left(T-t_{0}\right)},
$$

we can get

$$
\sup _{t_{0} \leq t \leq T} \mathbb{E}\left|x_{n}(t)\right|^{p} \leq\left(1+3^{p-1} \mathbb{E}\left|x_{0}\right|^{p}\right) e^{\beta\left(T-t_{0}\right)}=C .
$$


Lemma 2.3 Under the linear growth condition (6), for all $n \geq 1$ and $t_{0} \leq$ $s<t \leq T$ with $t-s \leq 1$ and $p \geq 2$,

$$
\mathbb{E}\left|x_{n}(t)-x_{n}(s)\right|^{p} \leq C_{p}(t-s)^{\frac{p}{2}}
$$

where $C_{p}$ is a positive constant related to $K, \quad p, T$.

Proof Note that

$$
x_{n}(t)-x_{n}(s)=\int_{s}^{t} f\left(\hat{x}_{n}(r), r\right) d r+\int_{s}^{t} g\left(\hat{x}_{n}(r), r\right) d B(r) .
$$

Hence, we can know that,

$$
\begin{aligned}
& \mathbb{E}\left|x_{n}(t)-x_{n}(s)\right|^{p} \\
\leq & \gamma \mathbb{E} \int_{s}^{t}\left[1+\left|\hat{x}_{n}(r)\right|^{2}\right]^{\frac{p}{2}} d r \leq 2^{\frac{p-2}{2}} \gamma \int_{s}^{t}\left[1+\sup _{s \leq u \leq r} \mathbb{E}\left|x_{n}(u)\right|^{p}\right] d r \\
\leq & \frac{1}{2}(8 K)^{\frac{p}{2}}\left(\frac{p(p-1)}{2}\right)^{\frac{p}{2}}(1+C)(t-s)^{\frac{p}{2}}=C_{p}(t-s)^{\frac{p}{2}},
\end{aligned}
$$

where

$$
\gamma=2^{p-1}(t-s)^{p-1} K^{\frac{p}{2}}+2^{p-1}\left(\frac{p(p-1)}{2}\right)^{\frac{p}{2}}(t-s)^{\frac{p-2}{2}} K^{\frac{p}{2}}
$$

and

$$
C_{p}=\frac{1}{2}(8 K)^{\frac{p}{2}}\left(\frac{p(p-1)}{2}\right)^{\frac{p}{2}}(1+C) .
$$

Lemma 2.4 [3, Theorem 7.1 in Section 1.7] Let $p \geq 2$. Let $g \in \mathcal{M}^{2}\left([0, T] ; R^{d \times m}\right)$ which denotes the family of $R^{d \times m}$-valued $\mathcal{F}_{t}$-adapted processes such that

$$
\mathbb{E} \int_{0}^{T}|g(s)|^{p} d s<\infty .
$$

Then

$$
\mathbb{E}\left|\int_{0}^{T} g(s) d B(s)\right|^{p} \leq\left(\frac{p(p-1)}{2}\right)^{p / 2} T^{\frac{p-2}{2}} \mathbb{E} \int_{0}^{T}|g(s)|^{p} d s .
$$

\subsection{Proofs of main results}

In this subsection, we start to prove our main theorems.

Proof [Proof of Theorem 2.1] Assume that the conclusion of the theorem is not true. Then there exist positive constants $\delta$ and $T$ such that

$$
\lim _{n \rightarrow \infty} \mathbb{E}\left(\sup _{t_{0} \leq t \leq T}\left|x(t)-x_{n}(t)\right|^{p}\right) \geq 2 \delta
$$


Without loss of any generality we may assume that for all $n$,

$$
\mathbb{E}\left(\sup _{t_{0} \leq t \leq T}\left|x(t)-x_{n}(t)\right|^{p}\right) \geq \delta .
$$

By Theorem 1.2 and Lemma 2.3, we know that the family of the processes

$$
\left\{x_{n}(t), \hat{x}_{n}(t), x(t), B(t)\right\}_{n \geq 1}
$$

are tight [1, Theorm 4.2 and Theorem 4.3]. Therefore, there exists some probability space $(\bar{\Omega}, \overline{\mathrm{F}}, \bar{P})$ and a sequence of stochastic processes $\left\{\bar{x}_{n}(t), \bar{y}_{n}(t), \bar{z}_{n}(t), \bar{B}_{n}(t)\right\}_{n \geq 1}$ on this probability space, which have the following properties:

1. For each $n \geq 1$, the probability law of $\left\{\bar{x}_{n}(t), \bar{y}_{n}(t), \bar{z}_{n}(t), \bar{B}_{n}(t)\right\}$ coincides with the law of $\left\{x_{n}(t), \hat{x}_{n}(t), x(t), B(t)\right\}$.

2. There exists a subsequence $\left\{n^{\prime}\right\}$, which may be assumed to be the same as $\{n\}$ without loss of generality, such that

$$
\left\{\bar{x}_{n}(t), \bar{y}_{n}(t), \bar{z}_{n}(t), \bar{B}_{n}(t)\right\} \rightarrow\left\{\bar{x}_{0}(t), \bar{y}_{0}(t), \bar{z}_{0}(t), \bar{B}_{0}(t)\right\}, \quad \bar{P}-\text { a.s. }
$$

uniformly on every finite time interval.

These two properties with inequality (12) imply immediately that

$$
\begin{aligned}
\delta & \leq \liminf _{n \rightarrow \infty} \mathbb{E}\left(\sup _{t_{0} \leq t \leq T}\left|x(t)-x_{n}(t)\right|^{p}\right) \\
& =\liminf _{n \rightarrow \infty} \overline{\mathbb{E}}\left(\sup _{t_{0} \leq t \leq T}\left|\bar{z}_{n}(t)-\bar{x}_{n}(t)\right|^{p}\right) \\
& =\overline{\mathbb{E}}\left(\sup _{t_{0} \leq t \leq T}\left|\bar{z}_{0}(t)-\bar{x}_{0}(t)\right|^{p}\right) .
\end{aligned}
$$

On the other hand, by the property (1) we have that for all $n \geq 1$,

$$
\bar{x}_{n}(t)=x_{0}+\int_{t_{0}}^{t} f\left(\bar{y}_{n}(s), s\right) d s+\int_{t_{0}}^{t} g\left(\bar{y}_{n}(s), s\right) d \bar{B}_{n}(s)
$$

and

$$
\bar{z}_{n}(t)=x_{0}+\int_{t_{0}}^{t} f\left(\bar{z}_{n}(s), s\right) d s+\int_{t_{0}}^{t} g\left(\bar{z}_{n}(s), s\right) d \bar{B}_{n}(s) .
$$

Letting $n \rightarrow \infty$, we find (see Skorohod [4]) that

$$
\bar{x}_{0}(t)=x_{0}+\int_{t_{0}}^{t} f\left(\bar{y}_{0}(s), s\right) d s+\int_{t_{0}}^{t} g\left(\bar{y}_{0}(s), s\right) d \bar{B}_{0}(s),
$$


and

$$
\bar{z}_{0}(t)=x_{0}+\int_{t_{0}}^{t} f\left(\bar{z}_{0}(s), s\right) d s+\int_{t_{0}}^{t} g\left(\bar{z}_{0}(s), s\right) d \bar{B}_{0}(s),
$$

Note by the property (1) and Lemma 2.3 that

$$
\overline{\mathbb{E}}\left|\bar{x}_{n}(t)-\bar{y}_{n}(t)\right|^{p}=\mathbb{E}\left|x_{n}(t)-\hat{x}_{n}(t)\right|^{p} \rightarrow 0 \text { as } n \rightarrow \infty,
$$

which together with the property $(2)$ implies that $\bar{x}_{0}(t)=\bar{y}_{0}(t) \bar{P}$-a.s. It then follows from the equation (14) that

$$
\bar{x}_{0}(t)=x_{0}+\int_{t_{0}}^{t} f\left(\bar{x}_{0}(s), s\right) d s+\int_{t_{0}}^{t} g\left(\bar{x}_{0}(s), s\right) d \bar{B}_{0}(s) .
$$

So we can see that both $\bar{x}_{0}(t)$ and $\bar{z}_{0}(t)$ are solutions of equation (1) with respect to the Brownian motion $\bar{B}_{0}(t)$ under the same initial condition. According to the uniqueness of solutions of stochastic differential equations, we get $\bar{x}_{0}(t)=\bar{z}_{0}(t)$ which contradicts inequality (12). So the conclusion of this theorem is true.

\section{References}

[1] N. Ikeda and S. Watanabe, Stochastic differential equations and diffusion processes. North-Holland Publishing Co., Amsterdam-New York; Kodansha, Ltd., Tokyo, 1981.

[2] X. R. Mao, Approximate solutions for stochastic differential equations with pathwise uniqueness. Stochastic Anal. Appl. 12 (1994), no. 3, 355367.

[3] X. R. Mao, Stochastic differential equations and their applications. Horwood Publishing Limited, Chichester, 1997.

[4] A. V. Skorokhod, Studies in the theory of random processes. AddisonWesley Publishing Co., Inc., Reading, Mass., 1965.

Received: June 1, 2014 